\title{
Brain regions associated with psychological pain
}

\author{
Astrid Habenstein - Thomas Reisch • Konrad Michel
}

Published online: 12 April 2013

(C) Springer Science+Business Media New York 2013

\section{To the Editor}

We read with great interest the overview of neural correlates of psychological pain by Meerwijk et al. (2012), a broad concept widely used in clinical practice. As the authors had noted in an earlier publication (Meerwijk and Weiss 2011), various concepts of psychological pain have been put forward, but so far, no commonly agreed definition has been established. From their review of the literature, Meerwijk et al. define psychological pain as "a lasting, unsustainable, and unpleasant feeling resulting from negative appraisal of an inability or deficiency of the self'. They state that "psychological pain is a lasting feeling in that it endures, it does not pass quickly, and it takes time to resolve". From our work with suicidal patients, we would like to argue that suicidal psychological pain as it was investigated in our study (Reisch et al. 2010) does not fit into this definition.

For their review, Meerwijk et al. only found two neuroimaging studies using an established measure of psychological, or mental, pain (OMMP, Orbach et al. 2003). In both studies, psychological pain was related to suicidality. Van Heeringen et al. (2010), in their patient sample, found an association between OMMP scores and suicidality. Reisch et al. (2010) related neural activation in suicide attempters to autobiographically recalled mental pain immediately preceding (triggering) an episode of suicidal self-harm.

A suicidal crisis is generally understood as an acute and time-limited form of psychological pain, characterized by physiological arousal and the feeling of unbearability (Rudd et al. 2004, p.30). The high levels of psychological pain have been stressed by Shneidman (1993), who argued that suicide occurs when a person's individually determined

A. Habenstein $\cdot$ T. Reisch $\cdot$ K. Michel $(\triangle)$

University Hospital of Psychiatry, Bern, Switzerland

e-mail: konrad.michel@spk.unibe.ch psychological pain threshold is exceeded. This is consistent with the association of suicide with dissociative symptoms (Orbach 1994), as an expression of high emotional arousal. In the OMMP validation study, Orbach et al. (2003) found feelings of irreversibility, loss of control, emptiness, emotional flooding, emotional freezing, and estrangement to be the main factors - symptoms that are usually associated with dissociative experiences. We therefore believe that suicidal psychological pain is not only acute and time-limited in nature, but is also an extreme form of pain, usually exceeding the individual pain threshold. Psychological models consistent with this notion have, for example, been formulated by Baumeister (1990), and Maltsberger (2004).

The patients examined in our fMRI study (Reisch et al. 2010) reported high levels of dissociation. Consistent with this, during recall of mental pain associated with the suicidal behavior, we found a striking similarity of brain activation with neural correlates of trauma reactivation known from PTSD studies (e.g. Lanius et al. 2001), in particular reduced activity in the left medial prefrontal cortex (BA 6, 10/11) and the anterior cingulate gyrus (BA 32). This is not surprising in view of the fact that the association of suicidal behavior with trauma is well established (Orbach 1994; Mann and Currier 2010; Bruffaerts et al. 2010).

Psychological pain is invariably associated with appraisal, which is a major factor moderating intensity and duration. Cognitive appraisal of psychological pain as uncontrollable and unsustainable may result in suicidal behavior as the only solution, while other forms of coping such as self-soothing, help-seeking, etc. will help to contain psychological pain and allow it to subside (Rudd et al. 2004, p.237). Interestingly, in our fMRI study, when patients were asked to recall how, as a response to intense mental pain, they planned and initiated an action of self-harm, we found increased activation in certain cortical areas. This finding is in line with the classical study by Ochsner et al. (2002), who showed that stressful 
emotional experiences are associated with prefrontal deactivation, while the use of cognitive coping strategies as a response to negative emotions is related to increased activation in prefrontal cortical areas. Appraisal mechanisms probably explain why some studies found decreased activity in the ventromedial PFC while others reported an increase of activity related to psychological pain.

To summarize, we understand psychological pain associated with suicidal crises as an acute and time-limited intensive emotional experience with the quality of a traumatic experience. We believe that psychological pain such as it occurs in grief or unhappiness represent different entities with different neurobiological correlates. Therefore, in our opinion, future neuroimaging studies will be best advised to focus on clearly defined and specific forms of psychological pain, to include the time dimension, and to apply a validated measuring instrument (such as the OMMP) of psychological pain.

Conflict of interest The authors state that there is no conflict of interest pertaining to the work of Meerwijk et al.

\section{References}

Baumeister, R. F. (1990). Suicide as escape from self. Psychological Review, 97(1), 90-113.

Bruffaerts, R., Demyttenaere, K., Borges, G., Haro, J. M., Chiu, W. T., et al. (2010). Childhood adversities as risk factors for onset and persistence of suicidal behaviour. The British Journal of Psychiatry, 197(1), 20-27.

Lanius, R., Williamson, P. C., Densmore, M., Boksman, K., Gupta, M. A., Neufeld, R. W., et al. (2001). Neural correlates of traumatic memories in posttraumatic stress disorder: a functional MRI investigation. The American Journal of Psychiatry, 158, 1920-1922.

Maltsberger, J. T. (2004). The descent into suicide. The International Journal of Psychoanalysis, 85, 653-668.

Mann, J. J., \& Currier, D. M. (2010). Stress, genetics and epigenetic effects on the neurobiology of suicidal behavior and depression. European Psychiatry, 25, 268-271.

Meerwijk, E. L., \& Weiss, S. J. (2011). Toward a unifying definition of psychological pain. Journal of Loss \& Trauma, 16(5), 402-412.

Meerwijk, E. L., Ford, J. M., \& Weiss, S. J. (2012). Brain regions associated with psychological pain: implications for a neural network and its relationship to physical pain. Brain Imaging and Behavior [Epub ahead of print].

Ochsner, K. N., Bunge, S. A., Gross, J. J., \& Gabrieli, J. D. (2002). Rethinking feelings: an FMRI study of the cognitive regulation of emotion. Journal of Cognitive Neuroscience, 14, 1215-1229.

Orbach, I. (1994). Dissociation, physical pain, and suicide: a hypothesis. Suicide \& Life-Threatening Behavior, 24(1), 68-79.

Orbach, I., Mikulincer, M., Sirota, P., \& Gilboa-Schechtman, E. (2003). Mental pain: a multidimensional operationalization and definition. Suicide \& Life-Threatening Behavior, 33, 219-230.

Reisch, T., Seifritz, E., Esposito, F., Wiest, R., Valach, L., \& Michel, K. (2010). An fMRI study on mental pain and suicidal behavior. Journal of Affective Disorders, 126, 321-325.

Rudd, M. D., Joiner, T. E., \& Rajab, H. (2004). Treating suicidal behavior (2nd ed.). New York: Guilford.

Shneidman, E. S. (1993). Suicide as a psychache. Journal of Nervous and Mental Diseases, 181(3), 145-147.

van Heeringen, K., Van den Abbeele, D., Vervaet, M., Soenen, L., \& Audenaert, K. (2010). The functional neuroanatomy of mental pain in depression. Psychiatry Research, 181, 141-144. 